\title{
Synthesis of Unconventional Dynamic Merge Metering Traffic Control for Work Zones
}

\author{
Heng Wei ${ }^{*}, 1$, Manojkumar Pavithran ${ }^{2}$, Ping Yi ${ }^{3}$, Qingyan (Ken) Yang ${ }^{4}$ and Qing-An Zeng ${ }^{5}$ \\ ${ }^{I}$ Department of Civil \& Environmental Engineering, 792 Rhodes Hall, P.O. Box 210071, University of Cincinnati, \\ Cincinnati, Ohio 45221, USA \\ ${ }^{2}$ Y.S. Mantri and Associates LLC, 325 N. Austin Dr. Suite \# 2, Chandler, AZ 85226, USA \\ ${ }^{3}$ Department of Civil Engineering, ASEC 213, University of Akron, Akron, Ohio 45221, USA \\ ${ }^{4}$ Iteris, Inc., 1120 East Long Lake, Suite 222, Troy, Michigan, 48085, USA \\ ${ }^{5}$ Department of Computer Science, P.O. Box 210030, University of Cincinnati, Cincinnati, Ohio 45221, USA
}

\begin{abstract}
This paper presents an unconventional approach for work-zone bottleneck traffic control through integrating the dynamic late merge with a merge metering via wireless communication at the downstream taper area of a work zone,

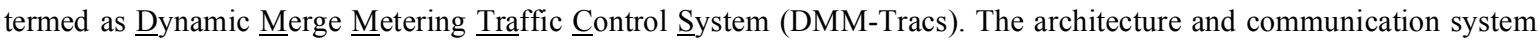
for the DMM-Tracs, including system components, communication technologies and operation mechanism, are introduced. With this system, the merge metering control will be activated depending on the volume detected at an upstream location of the work-zone bottleneck. If the thresholds of control parameters are met, a merge metering signal installed at the taper area will be activated and approaching vehicles will be informed of the metering situation through flashing signage or changeable message sign installed in the advance areas of the work zone. To reduce enforced merges and potentials of merge conflicts, the control scheme will be adjusted on the real-time basis depending on the detected traffic conditions. This potentially increases the efficiency with which the traffic is discharged into the open lane inside the work zone. A portable controller is the "brain" of the DMM-Tracs to receive data from detectors, process the data, and control the merge metering signal via wireless communications. The efficacy of the DMM-Tracs is evaluated via a microsimulation test bed with the state-of-the-art microscopic simulation software VISSIM. Meanwhile, the volume threshold values are also determined via the simulation. The simulation results indicate that higher lane-closure percentage of the bottleneck, more applicable the DMM-Tracs.
\end{abstract}

Keywords: Work zone, traffic control, dynamic merge metering, wireless communication, microscopic simulation.

\section{INTRODUCTION AND BACKGROUND}

It has been realized that in recent years, the focus of highway systems in many states has been shifted from building new transportation facilities to restoration, resurfacing, rehabilitation, and reconstruction of existing roadways, all of which give rise to scheduled construction activities at work zones. A work zone usually creates a bottleneck when one or more lanes in a multi-lane highway are closed to an accommodate construction activity in those lanes. Physical bottleneck is often a cause of congestion in a multi-lane highway usually because one or more lanes are closed to construction activity or incident. In addition to the traditional approach as specified in the Manual of Uniform Traffic Control Devices (MUTCD) using advance warnings and flaggers to control work zone traffic [1], several other traditional strategies have been developed to improve the flow of traffic at work zones. They aim to decrease congestion and reduce travel times and delay at the bottlenecks caused by work zones. These strategies include

*Address correspondence to this author at the Department of Civil \& Environmental Engineering, 792 Rhodes Hall, P.O. Box 210071, University of Cincinnati, Cincinnati, Ohio 45221, USA; Tel: 513-556-3781; Fax: 513556-2599; E-mail: weihg@ucmail.uc.edu static and dynamic versions of the early and late merge concepts, namely, 1) static early merge strategy, 2) dynamic early merge strategy, 3) late merge strategy, and 4) dynamic late merge (DLM) strategy. However, each of these strategies applies to a certain condition only, and none of them can perform well under varying traffic conditions, in particular under congestions [2].

Several field testing studies and literature reviews [2-4] indicate that, of the above strategies the late merge and dynamic late merge methods are better ones for traffic control for work zones. The late merge is designed to encourage drivers to use either the open or closed lane until they reach the merge point at the lane closure taper. In this method approximately 1.5 miles in advance of the lane closure, USE BOTH LANES TO MERGE POINT signs are placed on both sides of the roadway. These signs are followed by conventional ROAD WORK AHEAD and advance lane closed signs. Finally, MERGE HERE TAKE YOUR TURN signs are placed on both sides of the roadway near the beginning of the taper. The primary intent of the late merge is to reduce the road rage between early and late mergers by informing drivers that it is permissible for traffic to travel in both lanes to the merge point. A comparison 
study revealed that the conflict rates are substantially lower with the late merge and the capacity of the Late Merge can be nearly 20 percent higher than that of the conventional merge [4]. An evaluation conducted by Beacher et al recommended that late merge should be considered for 3-to1 lane-closure configuration only after a sound methodology for deployment has been developed and tested in the field [5]. For the 2-to-1 and 3-to-2 configurations, the Late Merge should be implemented only when the percentage of heavy vehicles is at least 20 percent.

DLM is intended to resolve the problems of earlier strategies, i.e., the increase in travel times in the early merge strategies and the potential for collisions in the late merge strategy. Ideally, it functions the conventional merge during periods of unsaturated flow, and switches to the late merge during congestions. However, most existing DLM systems that operate using static thresholds without real-time data cannot perform well under varying traffic conditions [6]. As a mater of fact, many challenges are faced with measuring travel time on a real-time basis, especially in terms of accuracy which often vibrates due to varied driver behaviors and technologies.

To overcome such a weakness of the DLM and other traditional and advanced strategies, this paper presents an unconventional alternative to traffic control at work zones. The method, termed as Dynamic Merge Metering Traffic Control System for work zones (DMM-Tracs), integrates the DLM with a merge metering at the taper area of a work zone. The DMM-Tracs provides an unconventional alternative to the DLM for increasing the efficacy of mitigating congestion at physical bottlenecks under varying traffic conditions. For the detection of traffic volume and speed is quite reliable in technology, volume and/or speed could be used as parameters to identify the traffic conditions. If the thresholds of control parameters are met, a merge metering signal installed at the taper area will be activated and an advance warning will be shown on a static sign with flashing on or a changeable message sign (CMS). To reduce enforced merges and potentials of merge conflicts, the control scheme will be adjusted on a real-time basis depending on the detected traffic conditions. A portable controller is the system "brain" to receive detected data from detectors, process the data, and control the merge metering system via wireless communications.

This paper is organized as follows. The architecture and communication system for the DMM-Tracs, including system components, communication technologies and operation mechanism are introduced. To evaluate the performance of DMM, simulation-based trials were conducted with the state-of-the-art microscopic simulation software VISSIM to compare the operations of DMM-Tracs and later merge control strategies, which also results in the volume threshold values.

\section{DEVELOPMENT OF DMM-TRACS SYSTEM}

\section{Layout of DMM-Tracs System}

The layout of the DMM-Tracs system consists of the following areas:

- $\quad$ Advance warning \& detection area and devices;
- Transition Area, including acceleration length and merging taper;

- $\quad$ Longitudinal buffer;

- $\quad$ Activity area;

- $\quad$ Termination area - downstream flare;

Advance Warning \& Detection Area and Devices: The MUTCD defines the advance warning area as the section of highway where road users are informed about the upcoming work zone or incident area. Per MUTCD standards warning is provided through a series of road signs. These signs warn the drivers of road work ahead, the lanes that are closed, the distance till the beginning of the road work and the extent of the road work.

In the DMM_Tracs system the signs perform the function of both warning the drivers about upcoming work zone and regulating the traffic entering the work zone. These functions are achieved through the use of a series of static and changeable message signs. The first sign encountered by the drivers is a temporary traffic control (TTC) warning sign "Road Work Ahead" at a distance of over 3 miles from the merge metering signal. It is followed by a system of three changeable message signs (CMS) at distances of 3 miles, 1 mile and $1 / 2$ mile from the merge metering signal. When metering is in progress the first CMS in the series encountered by the driver displays the message "ROAD WORK-3 MILES AHEAD-USE BOTH LANES". The next CMS displays the message "USE BOTH LANES-TILL SIGNAL-1 MILE AHEAD". The last CMS in the series displays the message "PREPARE TO STOP-AT SIGNALPROCEED ON GREEN". When metering is not in progress the CMS displays the message "RIGHT (LEFT) LANE CLOSED, X FEET(MILES) AHEAD".

Traffic sensors are provided at appropriate locations in the advance warning and detection area to monitor the volume and speed of the vehicles upstream of the traffic zone. When the detected traffic reaches threshold values of volume and speed the metering system is activated. This series of CMS is followed by the TTC warning sign "Right/Left Lane Closed" along with a supplementary plaque showing the distance " $1000 \mathrm{ft}$ " or the appropriate distance. This is followed by a TTC sign "Prepare To Stop When Flashing" on which a radio controlled high-intensity flashing light is mounted. This sign is placed at a distance that meets adequate advance placement of warning signs requirements per Table 2C-4 of the MUTCD.

Temporary portable traffic control signals are provided at a distance equal to the acceleration length before the beginning of the transition taper. At the location of the metering signal a regulatory sign R10-6 "Stop Here on RED' is provided. The pavement is striped with a temporary 24 " solid white pavement marking per section 6F.72 of the MUTCD, as a stop bar at the location where the vehicles have to wait for the signal.

Transition Area: the merging taper is provided as per conventional work zone layout designed per Table $6 \mathrm{H}-4$ of the MUTCD. The acceleration length included in this area is provided so as to enable the driver to gain the appropriate speed before merging into the open lane. This acceleration lane will potentially improve the efficiency with which 
vehicles are released from the merge metering signal. As such there does not exist a need for the drivers to position themselves in gaps in a through-traffic stream. Therefore, a length of $300 \mathrm{ft}$ which is the minimum gap acceptance length ' $\mathrm{L}_{\mathrm{g}}$ ' as specified in The Policy on Geometric Design of Highways and Streets published by the American Association of State Highway \& Transportation Officials (AASHTO) will be sufficient for the acceleration length. The acceleration length is striped with a temporary 8 " solid white pavement marking per section 6F.72 of the MUTCD, to maintain a clear separation of lanes till the taper.

Longitudinal Buffer: A longitudinal buffer is provided to create a recovery zone for errant vehicles. The Missouri Department of Transportation Engineering Policy Guide suggests a longitudinal buffer ranging from $120 \mathrm{ft}$ for speeds of $35 \mathrm{mph}$ to 550 for speeds between 60 to $70 \mathrm{mph}$.

Activity Area: The activity area represents the actual work area on the roadway. This activity area could include a downstream buffer area that could also be used for storage of construction equipment. A TTC warning sign W20-1 "Road Work ' $\mathrm{X}$ ' Miles" is placed at the beginning of the activity area.

Termination Area - Downstream Flare: The downstream flare is provided to help the drivers merge back into the unobstructed roadway. The MUTCD recommends a maximum flare of $100 \mathrm{ft}$ for the downstream flare. TTC warning sign G20-2 "End Road Work" is placed after the downstream flare.

The layout of the DMM-Tracs system is thus designed to warn the drivers of an approaching work zone, instruct them to stay on their respective lanes till the metering signal, regulate the discharge of vehicles into the work zone through the metering process and get them back into the unobstructed roadway at the end of the work zone. Fig. (1) illustrates the communication architecture of the DMM-Tracs. The wireless-communication-enhanced components of DMMTracs include:

- $\quad$ Merge metering signal including Fixed cycle Merge Metering (FMM) and Continuous Merge Metering (CMM). They are usually red and green signals, or traditional three-color signals;

- Portable roadside detectors measure traffic volume passing across a specified cross section of the roadway.. They are installed on the roadside (or on the pavement) and designated to cause minimal disruption to normal traffic operations. They are usually mounted on overhead structures or to the side of the pavement. There are various technologies applied for such a kind of detector, such as passive/active infrared, microwave, radar, ultrasonic, passive acoustic sensors, and video detectors. To detect throughput of the work zone, the other portable roadside detectors is installed in the work zone near the taper area;

- Portable controller is the system "brain" to receive detected data from detectors, process the data, and control the merge metering system via wireless communications;
- $\quad$ Portable CMS that can be controlled by the portable controller based on the detected traffic conditions; and

- Wireless communications components that link the portable controller with portable roadside detectors and portable changeable message signs.

\section{Wireless Communication System for DMM-Tracs}

The wireless communication technologies play a critical role for the integration and workout of all communicationenhanced components for the DMM-Tracs. The wireless communications of the DMM-Tracs includes the following integrations:

- Controller-to-detector communications: the wireless communication, data transmission, and command activation between the portable controller and portable roadside detectors; and

- Controller-to-CMS communications: the wireless communication, data transmission, and command activation between the portable controller and the changeable message signs as well as the flashing function of "DO NOT PASS WHEN FLASHING" signs.

- The primary requirements for DMM-Tracs communications include:

- The controller should be able to communicate with multiple road-side devices, including portable detectors, portable changeable message signs, and flashboards. This type of communication from the controller to road-side devices is termed as Point-toMultipoint (P2M) communication;

- The non-video data transmission capacity required for detectors and CMS is about $20 \mathrm{Kbps}$ or less;

- $\quad$ The wireless communication components should be flexible for easy and rapid deployment, and

- $\quad$ The wireless network should be cost-effective.

- $\quad$ As an option, if the DMM-Tracs system needs to be tied with TMC (Traffic Management Center), then an additional Point-to-Point (P2P) communications link should be included.

The wireless communication performance requirements for DMM-Trac system are as follows:

- Architecture: P2M for the communication of controller and other devices;

- Working frequency band: unlicensed bands;

- Access technology: FDMA (Frequency Division Multiple Access), TDMA (Time Division Multiple Access), CDMA (Code Division Multiple Access), or OFDM (Orthogonal Frequency Division Multiplexing)

- $\quad$ Actual throughput: $>40 \mathrm{Kbps}$ (assumption of $50 \%$ overhead for non-video data);

- $\quad$ Coverage: 3-4 miles; and

- $\quad$ Outdoor units 


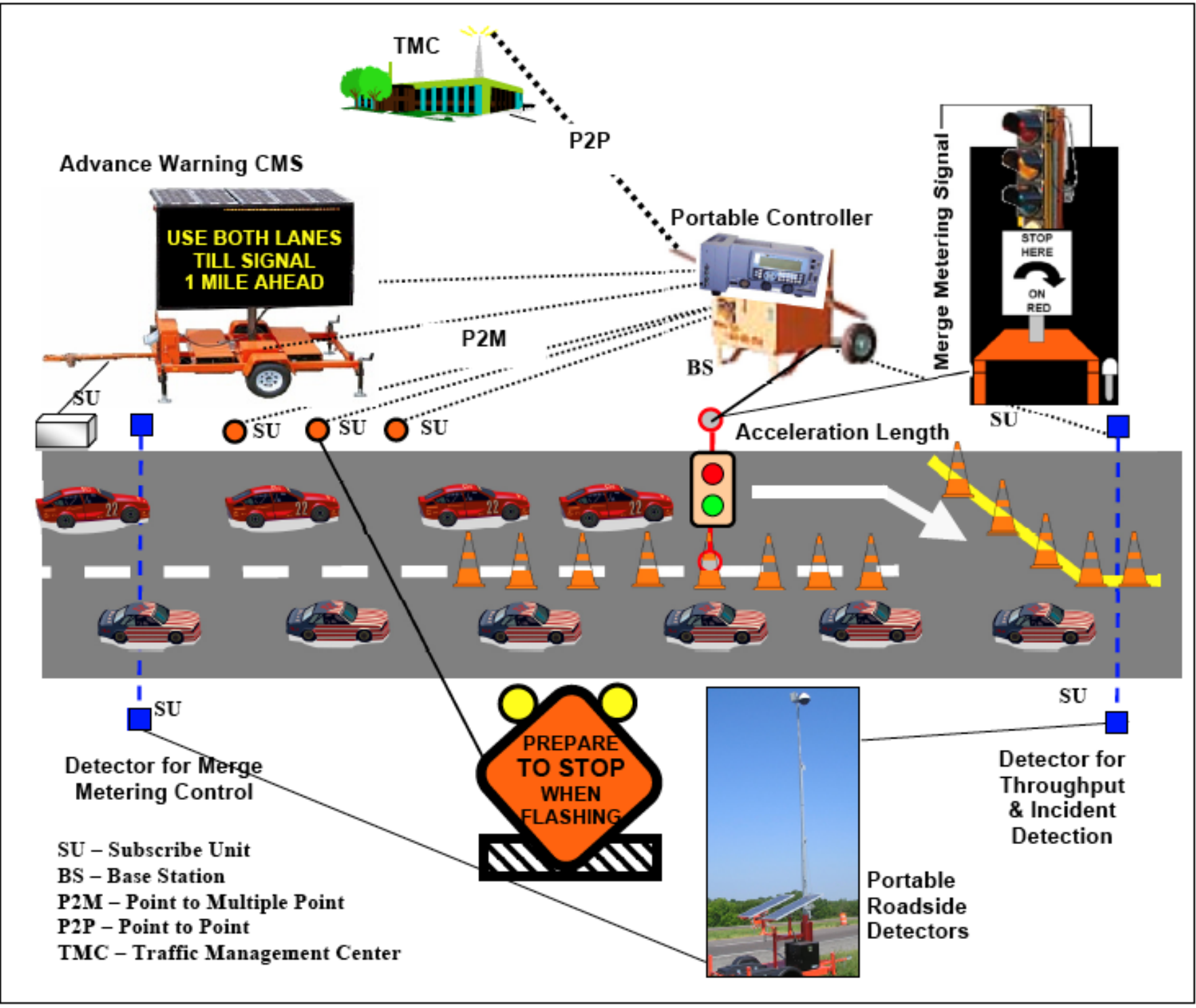

Fig. (1). Wireless-enhanced components.

Currently, there are many wireless communications technologies available for data transmission, from cell phone technologies (e.g., GPRS for GSM network and 1xRTT for CDMA network), to traditional Spread Spectrum radio, as well as Broadband Wireless Access (BWA) technologies (e.g., wireless LAN (802.11x), and industrial BWA). BWA belongs to "Fixed Wireless Communication" technology that uses "Spread Spectrum" technology and various modulations, media access and coding technology to support Ethernet based data communications at line-of-sight (LOS) or non-line-of-sight (NLOS) range. The spectrum bands available for these broadband wireless technologies include the unlicensed bands at $900 \mathrm{MHz}, 2.4 \mathrm{GHz}$ and $5.8 \mathrm{GHz}$, as well as the licensed bands at $2.3 \mathrm{GHz}, 2.5 \mathrm{GHz}$, and $4.9 \mathrm{GHz}$ (public safety band). BWA technology has been widely used today in many enterprise applications, such as ISP backbone connection, public "Wi-Fi" network, remote security and surveillance, and ITS applications. Generally, there are two classes of BWA products available on the current market.
- IEEE 802.11x based wireless Ethernet ("Wi-Fi") products; and

- Industrial wireless Ethernet products;

The candidate products for $\mathrm{P} 2 \mathrm{M}$ wireless communication should be selected from existing industrial unlicensed band BWA products. The following benefits of BWA technology could be considered in designing the P2P and P2M communication systems for DMM-Tracs:

- $\quad$ Very high throughput (maximum up to $300 \mathrm{Mbps}$ );

- Low latency, always-on connection;

- Flexibility in service delivery using shared or dedicated bandwidth;

- $\quad$ Fast, easy deployment;

- Affordable, lower total cost of ownership;

- Unregulated frequency option - unlicensed frequency band; and 
- $\quad$ Outdoor environment tolerance.

The actual data throughput for a P2M BWA communication link is not only determined by the product specification, but also related to the communication range and the amount of actual subscribe units (communication links) for each base station. Generally, most of current industrial BWA products meet the requirements for this type of non-video data communications. Under this configuration, the base station for DMM-Tracs will be placed together with the controller by wire-line communication. Each subscribe unit will be tied with each portable detector, portable changeable message sign, and sign flashboard.

If the communication with the TMC (Traffic Management Center) is needed, the $\mathrm{P} 2 \mathrm{P}$ communication should be setup between the controller and the TMC. Most of the $\mathrm{P} 2 \mathrm{P}$ products could provide more than $10 \mathrm{Mbps}$ throughput at a LOS or NLOS range. Therefore, the existing P2P industrial BWA devices are powerful enough to handle the non-video data transmission between the DMM-Tracs and TMC.

Furthermore, if the video stream transmission is needed for $\mathrm{P} 2 \mathrm{P}$ communication, the $\mathrm{P} 2 \mathrm{P}$ throughput capacity is determined by the format of the video stream itself (analog NTSC, MJPEG, MPEG, and MPEG4, etc.), the frame rate of the video stream (full motion -- 24-30 frames per second, or slow scanned video -- less than 24 frames per second). And in the meanwhile, it also is related to the amount of video streams that need to be transmitted simultaneously. The P2P throughput should be at least 3 Mbps.

Finally, the following issues should be carefully considered when selecting suitable industrial BWA devices:

- $\quad$ Cost of products;

- Availability of the products, maturity of the technology;

- Maintainability, such as sufficient support service available from the manufacturer; and

- If the P2P link is needed, for easy maintenance reason, it is desirable to have the $\mathrm{P} 2 \mathrm{P}$ and $\mathrm{P} 2 \mathrm{M}$ products from the same manufacturer so that minimum integration effort would be needed in design and implementation.

\section{Concept of Operation for DMM-Tracs}

The concept of operation briefly describes the mechanism of the operation process of the DMM-Tracs for a scenario where co-coordinating the traffic flows through a bottleneck section at a highway work zone due to construction.

Consider the scenario with one lane closure at the right lane. Traffic enters the work zone from the left. In the DMM-Tracs, portable detectors are placed about $1.5-2$ miles ahead the taper area of the work zone and a place within the work zone, respectively. The portable detectors detect the passages and speeds of the vehicles and send the detected data to the portable controller at a time interval (e.g., 20 seconds). The controller aggregates the data into applicable flow rates (e.g., vehicles per hour or per 5 or 15 minutes) and average speed on a real-time basis. Based on the transmitted volume and/or speed data from the above detectors, the algorithm embedded within the controller will automatically make a decision whether to send an "activation command" to turn on the flashing function of the road-side sign flashboards.

Traffic sign flashboards placed between the detectors and the merge metering signal inform the drivers about the bottleneck ahead and convey the recommended speed limit. If the traffic is quite heavy, there would be quite many vehicles in the closed lane near the bottleneck portion that need forced merges switching onto the adjacent open lane. In this case the traffic flashboards recommend a slower approach speed for all incoming vehicles. Contrarily, if the traffic is light, flashboards advertise a relatively higher approach speed to enable the vehicles to utilize the lighttraffic-loaded lane and pass the bottleneck faster.

With increase of upstream traffic, the open lane(s) and closed lane(s) become more closely busy, and more forced merges would happen in the taper area or in close proximity to it. As a result, more delays and longer queues would be caused in the section ahead of the bottleneck, and the potential of the collision would become highly possible. The merge metering signal is thus applied here to control the traffic approaching the work zone, especially the traffic of the closed lane, as well as to minimize the possibility of accident when the traffic is heavy. Merge metering signal is considered to include fixed cycle merge metering (FMM) and continuous merge metering (CMM), which are explained in details below. They are usually red and green signals only with CMM, or traditional three-color signals with FMM. If the detected volume exceeds the threshold(s), the merge metering signal installed before the taper areas of the work zone will be automatically activated by the controller. The traffic will follow the merge metering signal control while approaching the work zone. The duration of implementing the merge metering signal depends on the comparison of the volumes (throughput) and speeds that are detected by the portable detectors installed ahead and within the work zone.

\section{Fixed Cycle Merge Metering (FMM)}

The fixed cycle merge metering strategy consists of fixed cycle lengths such as cycles of 30 seconds, 60 seconds, or 120 seconds each, depending on the traffic conditions. These cycle lengths consist of green, amber and red intervals just like a signal at any intersection. For evaluation purpose, in this study the green interval is equally divided among all lanes as the traffic volumes have been coded as equal in both lanes. However, in actual conditions the traffic volume distribution is not always equal on all lanes. Therefore, the green interval duration could possible be divided in the same ratio as the lane distribution.

\section{Continuous Merge Metering (CMM)}

The continuous merge metering strategy is similar to the ramp metering strategy in approach. Here the vehicles in each lane have alternating green and red signals for one second each. The alternating green and red signals are displayed continuously, throughout the metering operation. This approach gives motorists of each lane a short duration of opportunity to proceed unobstructed into the open lane. 
Control thresholds for activating the DMM-Tracs are potentially identified as multiple parameters, which may include traffic demand and throughput at work zone, upstream speed and work-zone speed, occupancy and so forth. It has been commonly recognized that the traffic volume is the most reliable data that could be obtained from the portable roadside detectors [7]. And the authors have initially identified that the merge metering signal will be activated when the traffic is under the congested conditions. The threshold of traffic demand, or arrival traffic at the upstream area of the work zone, is one of the critical control thresholds for activating merge metering signal. As the first step in studying the control parameters and developing the DMM-Tracs algorithm, three scenarios of lane-closure configurations at work zone are studied to identify the threshold values of traffic demand for activating the merge metering signal. The three scenarios are illustrated by Fig. (2), including 2-to-1, 3-to-1, and 3-to-2 lane-closure configurations. 2-to-1 is referred to the case where one lane is closed for the sake of work-zone construction in a highway with two lanes per direction. And 3-to-1 and 3-to-2 lane-closures are referred to the cases where one lane and two lanes remain at the work zone section of a highway with three lanes per direction, respectively. At the initial study stage, the volume threshold is determined using the simulation-based method.

\section{SIMULATION-BASED DETERMINATIONS OF CONTROL THRESHOLDS}

\section{Building VISSIM Simulation Test Bed}

To evaluate the effect of the merge metering strategy for traffic control at high-speed multi-lane highway or frewway work zones, in particular to determine thresholds of control parameters that can be used in the algorithm embedded within the DMM-Tracs. In this study, volume thresholds under varied configurations of lane closure, and delays $v s$ volumes for varied lane-closure configurations are studied.

Microscopic simulation software VISSIM is used to build the simulation-based test bed. VISSIM is coded to measure the travel times and delays for this section for all scenarios. After validation of the simulation test bed with observed data, the work zone is simulated for different volumes and percentages of heavy vehicles to obtain comparative performances for scenarios with 2-to-1, 3-to-1, and 3-to-2 configurations, respectively, as shown in Fig. (2) 80 runs for each scenarios are conducted. VAP language is used to code special metering schemes.

Fig. (3) shows real work zones at I-71 and McMillan Street in Cincinnati, Ohio. Observed speed ranges and merge behaviors are gained from those two sites for validating the simulation test bed.

As an example, Fig. (4) shows the layout of the VISSIM simulation model for the scenario of 2-to-1 lane closure. In Dynamic Merge Metering method, vehicles are encouraged to drive up to the merge meter at the merge point and wait for the green signal for their lane to move into the open lane. On green the vehicles can freely move into the open lane as vehicles in the other lane will be stopped for the red signal. For the Fixed Cycle Merge Metering strategy, the network is simulated for three cycle lengths of 30,60 and 120 seconds each as the metering signal plan. This cycle is split equally between the two lanes with green time of 13, 28 and 58 seconds, respectively. The best performance among all the three cycle lengths is chosen for the comparison.

As the upstream volume exceeds the capacity of the work zone, queues are observed to form at the merge point. The speeds at the merge point are found to be between 5 and 10 $\mathrm{mph}$. For speeds of $10 \mathrm{mph}$ the amber phase required to bring the vehicles to a complete stop is 1.73 seconds as calculated from the formula:

$Y=t+\frac{1.467 v}{2(a+64.4 s)}$

where

$Y=$ amber time required,

$t=$ perception time, second,

$v=$ speed, $10 \mathrm{mph}$

$a=$ deceleration rate, $\mathrm{ft} / \mathrm{sec}^{2}$,

$s=$ slope $/$ grade.

The amber phase is two seconds for queuing conditions. For free flow conditions, 85 -percentile speed is assumed to be $65 \mathrm{mph}$. The amber time using the Equation (1) is calculated as 5.6 seconds. Therefore, for volumes exceeding the capacity the amber time is kept at 2 seconds, and for volumes below the capacity the amber time is kept at 6 seconds. Unlike any signal control schemes for an intersection, vehicles are not required to complete lengthy turning manoeuvres to clear the intersection before the other movement is given green time. Therefore, an all-red phase is not required for the merge metering signal plan.

The merging maneuvers for the CMM and FMM methods are simulated using the priority rules option provided in VISSIM. The minimum gap acceptance was set at 0.0 seconds as the vehicles will be waiting at the red stop line and do not need to search for gaps. The vehicles have to look for acceptable headway ahead of the stop line. The metering is set up to be one-second green and one-second red for both lanes alternately.

The Late Merging maneuver is simulated using the priority rules option in VISSIM. The minimum gap acceptance was set at 2.2 seconds. The default value in VISSIM is 3.0 seconds. Under work zone conditions, it is observed that the drivers accept smaller gaps for lane change and complete the whole maneuver in a shorter time as compared to normal driving conditions [8]. The minimum spacing of the platoon is set to $19.0 \mathrm{ft}$.

\section{Analysis of Simulation Results}

Based on the simulation results, Fig. (5) shows a comparative graph for the delays of the three methods, namely, late merge, dynamic merge metering with FMM, and dynamic merge metering with CMM for the 2-to-1 configuration.

This result indicates that dynamic merge metering strategies with both FMM and CMM begin to perform better 


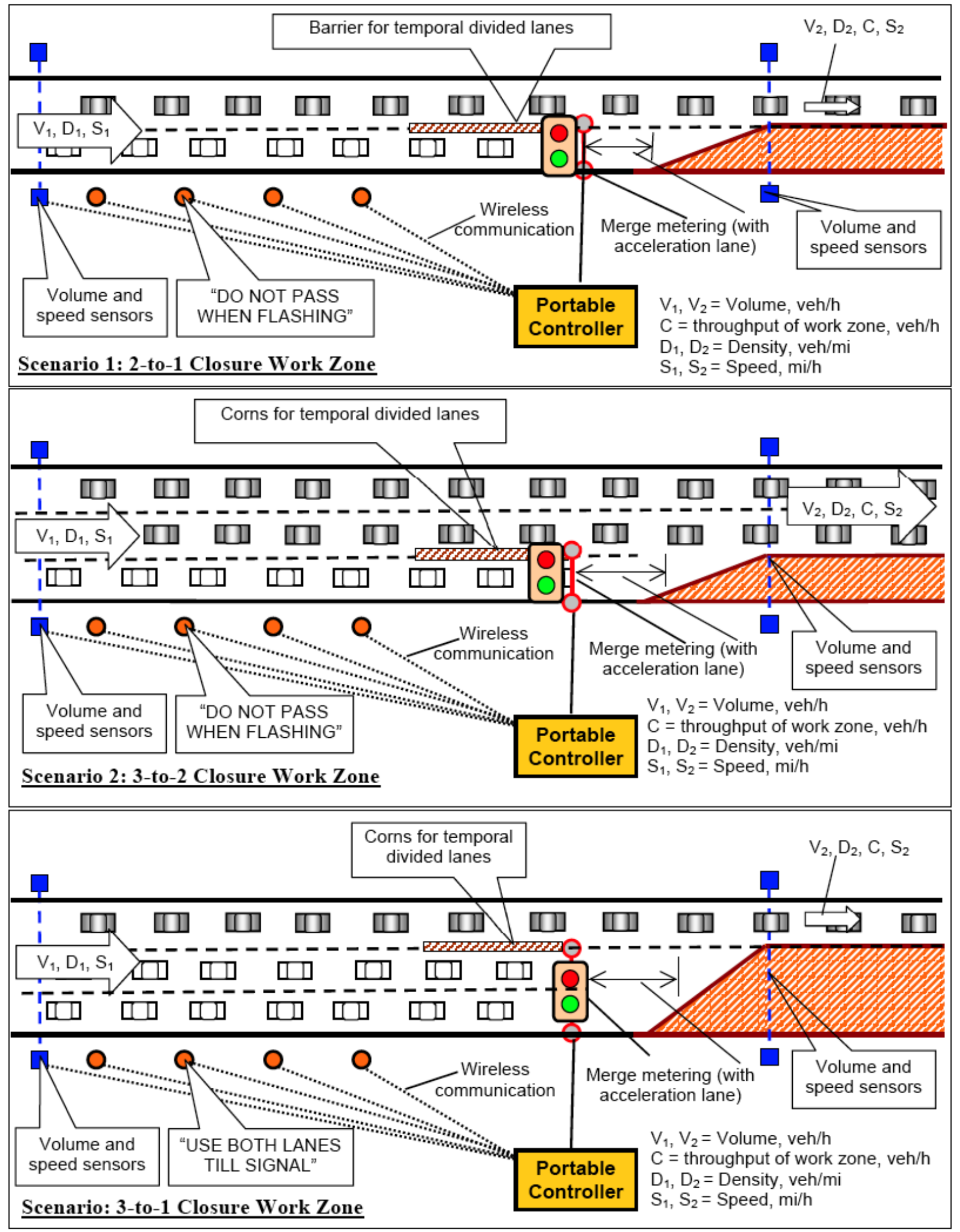

Fig. (2). Three cases of lane-closure patterns at work zone using DMM-Tracs. 


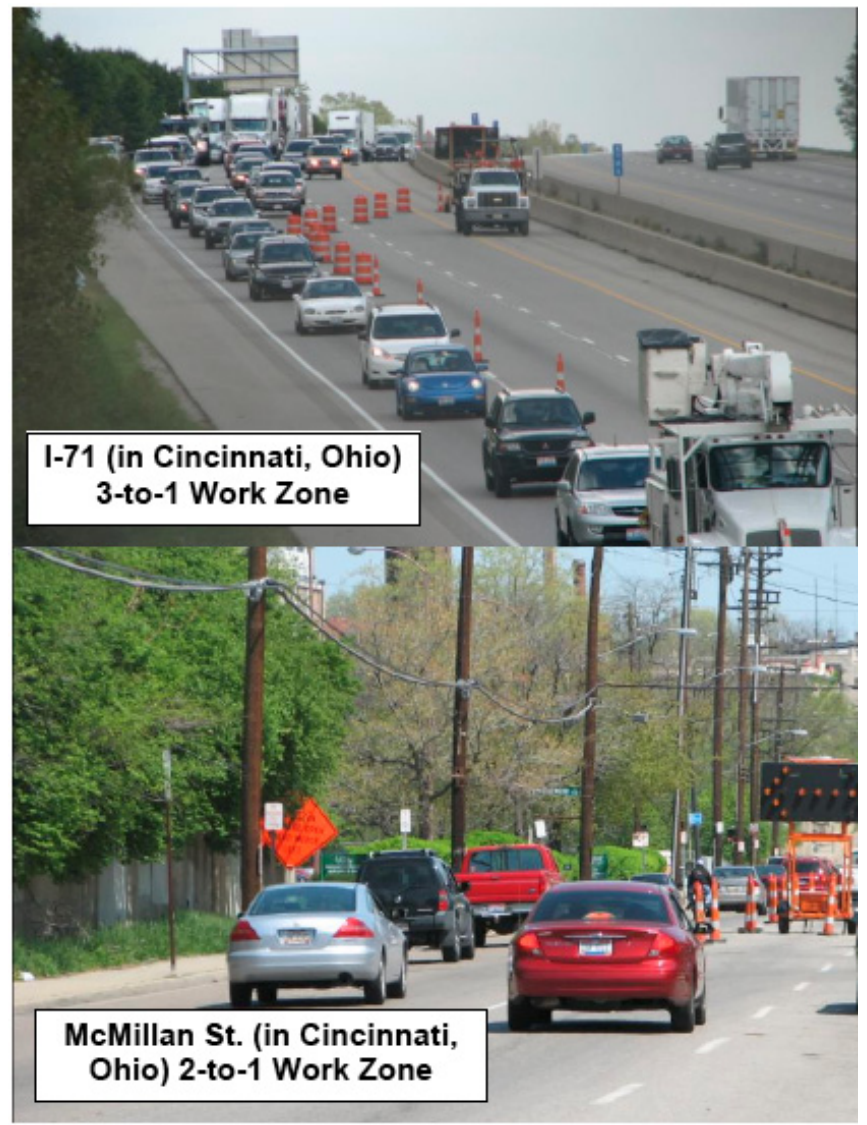

Fig. (3). Observation sites for simulation validation.

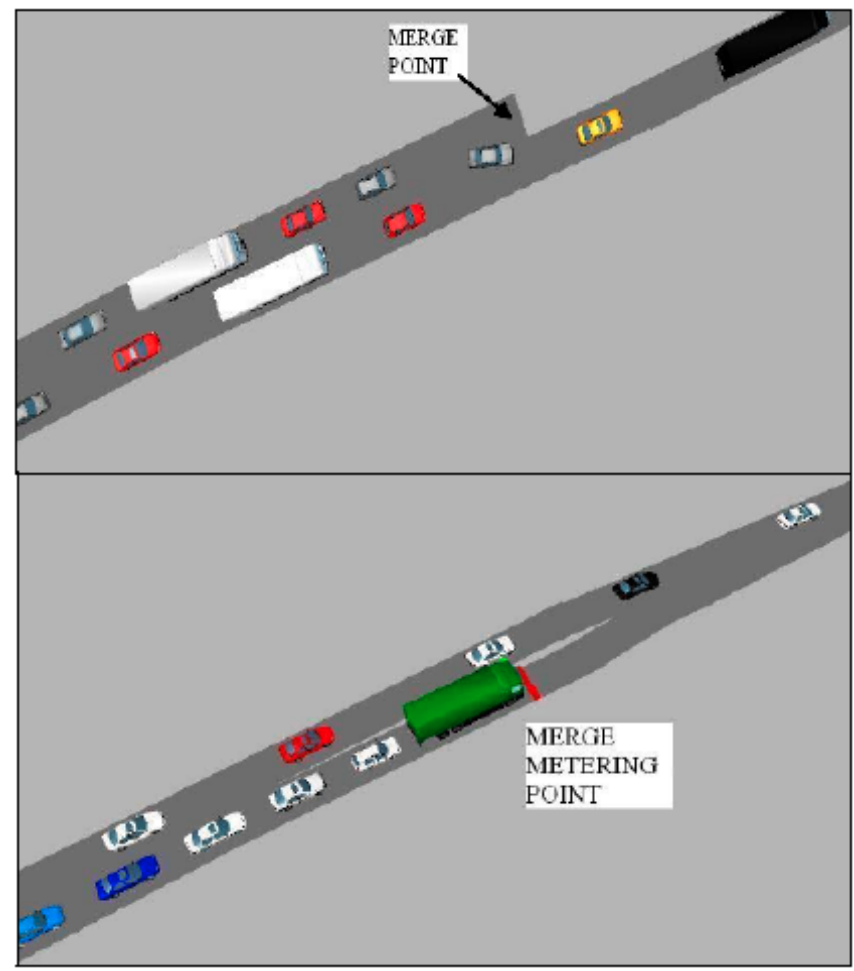

Fig. (4). Screen shot of VISSIM simulation with DMM and later merge (Scenario 1). 2-to-1 Scenario of Late Merge vs. Dynamic Merge Metering (Delay vs. Volume)

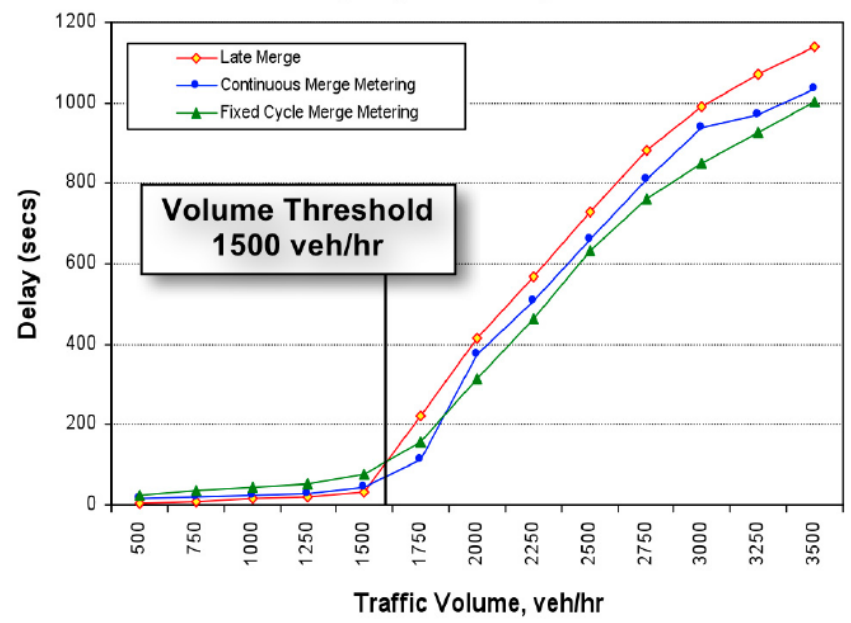

3-to-1 Scenario of Late Merge vs. Dynamic Merge Metering (Delay vs. Volume)

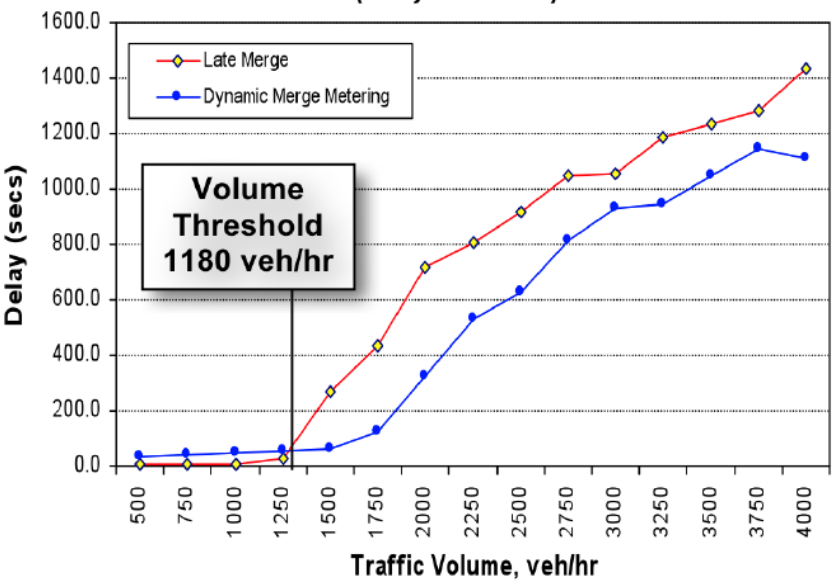

3-to-2 Scenario of Late Merge vs. Dynamic Merge Metering (Delays vs. Volume)

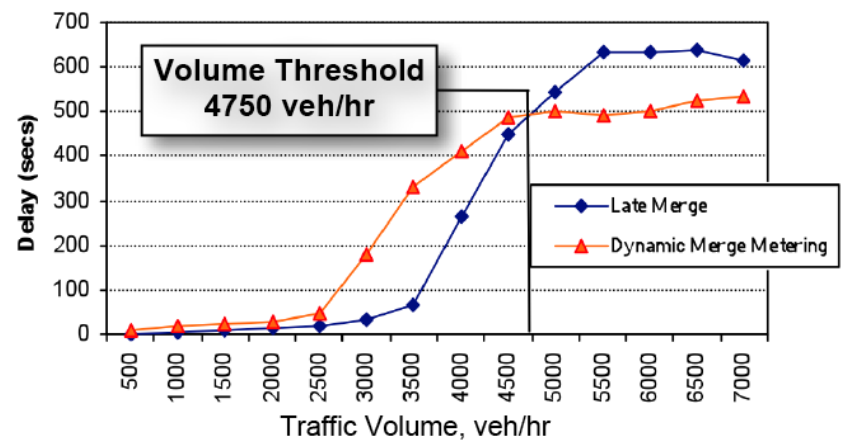

Fig. (5). Simulation results of delay $v s$ volume for three scenarios.

than the late merge as the volume exceeds $1,600 \mathrm{vph}$, which approximately coincides with the capacity of a freeway work zone with a 2-to-1 lane-closure configuration [9]. It is also observed from the simulation results that the Dynamic Merge Metering strategy discharges queues at a faster rate than the late merge strategy, thus produce shorter delays. The average reductions in delay for cases where all upstream 
volumes exceeds the work zone capacity are $21.30 \%$ using the FMM method and approximately $20.00 \%$ using the CMM method, respectively. Similarly, the average reductions in travel times are $11.46 \%$ using the FMM method and approximately $8.00 \%$ using the CMM method, respectively. Accordingly, the value of 1,600 vph could be used as the volume threshold to activate the merge metering signal for the scenario with 2-to-1 configuration (i.e., scenario 1).

Similarly, Fig. (5) also shows the comparative simulation results for the delays of scenarios with 3-to-1 and 3-to-2 lane-closure configurations. It can be observed that the volume threshold is $1,180 \mathrm{vph}$ for scenario 3 -to-1; 4,750 vph for scenario 3-to-2. Thus, all discussed volume thresholds are summarized by Table 1 .

Table 1. Simulation Results of the Volume Thresholds

\begin{tabular}{|c|c|c|}
\hline $\begin{array}{c}\text { Lane-Closure } \\
\text { Scenarios }\end{array}$ & $\begin{array}{c}\text { Closure } \\
\text { Percentage }\end{array}$ & $\begin{array}{c}\text { Volume Threshold for } \\
\text { Merge Metering Signal }\end{array}$ \\
\hline \hline 3-to-2 (1 of 3 lanes closed) & $33.3 \%$ & $4,750 \mathrm{vph}$ \\
\hline 2-to-1 (1 of 2 lanes closed) & $50.0 \%$ & $1600 \mathrm{vph}$ \\
\hline 3-to-1 (2 of 3 lanes closed) & $66.7 \%$ & $1,180 \mathrm{vph}$ \\
\hline
\end{tabular}

If the closure percentage is defined as the percentage of the number of closed lanes at the bottleneck over the total number of upstream lanes, it can be seen from the above result that higher the closure percentage, lower volume threshold at which the DMM-Tracs is required to be activated. In other words, higher closure percentage, more applicable the DMM-Tracs.

Additionally, the simulation results of the scenario with 3-to-1 configuration indicate that the maximum throughput at the work zone appears to be between 1250 and $1500 \mathrm{vph}$. The capacity of the 3-to-2 configuration appears to be approximately $3500-4000 \mathrm{vph}$. The speed drops and density suddenly increases after $4500 \mathrm{vph}$.

Regarding the two options included in the scenario with 3-to-2 configuration, it is interesting to note that the option 2 works much better than option 1 in reducing delay, which means that it is better to meter all lanes than just the closed lane. But more investigations are needed to verify this conclusion.

\section{CONCLUSIONS}

The DMM-Tracs system has been developed at a high level as an unconventional alternative to congestion and safety problems at a heavy traffic highway bottleneck that is caused by a long-period construction. Its architecture and configuration of the communication system is presented in this paper, and some applied communication technologies are also recommended. One of major advantages of the
DMM-Tracs over other work-zone traffic control strategies lies in the capability of switching various control options in response to varied traffic conditions. As mentioned earlier, there may be multiple contributing factors that should be considered in the algorithm for controlling the DMM-Tracs system. Only volume threshold for activating the merge metering signal is studied through building the VISSIM simulation-based test bed. The study of all other potential control parameters for DMM-Tracs is underway, including speed, density and occupancy, and the algorithm for controlling DMM-Tracs is also under the development.

In the research presented in this paper, three scenarios of lane closures at a work-zone bottleneck are studied in order to evaluate the effect of Dynamic Merge Metering strategy and determine the volume threshold for activating the merge metering signal. For the 2-to-1 lane-closure configuration, all simulations runs for the merge metering method are done for a signal cycle of 30,60 and 120 seconds. These may not include the most optimum cycle lengths for all traffic volumes, but the results show that 60 and 120 second cycles perform well in all cases whereas a 30 second cycle is inadequate to handle high volumes. The optimum cycle length may depend on the operating speeds, volume and traffic composition at each location. Further research is needed to find out the optimum cycle length for each location.

\section{REFERENCES}

[1] FHWA (Federal Highway Administration). Manual on Uniform Traffic Control Devices (MUTCD), 2003 Ed.. Washington D.C., 2003.

[2] R.M. Mousa, N.M. Rouphail and F. Azadivar. "Integrating Microscopic Simulation and Optimization: Application to Freeway Work Zone Traffic Control," in Transportation Research Record: Journal of the Transportation Research Board, No. 1254. TRB, National Research Council, Washington, D.C., 1990, pp 14-25.

[3] P.T. McCoy, G. Pesti, and P.S. Byrd. Alternative Information to Alleviate Work Zone-Related Delays: Final Report. NDOR Research Project No. SPR-PL-1(35) P513, Nebraska Department of Roads, Lincoln, Nebraska, February 1999.

[4] A. Tarko, S. Kanipakapatman, and J. Wasson. Modeling and Optimization of the Indiana Lane Merge Control System on Approaches to Freeway Work Zones, Final Report. FHWA/IN/JTRP-97/12, Purdue University, West Lafayette, Indiana, 1998.

[5] A.G. Beacher, M.D. Fontaine, and N.J. Garber. Evaluation of the Late Merge Work Zone Traffic Control Strategy. VTRC 05-R6, Final Report to Virginia Transportation Research Council, Charlottesville, Virginia, 2005.

[6] K.P. Kang, and G.L. Chang. Development of an Advanced Dynamic Late Merge Control Model and Algorithm (Paper \#061183). Compendium of Papers CD-ROM, $85^{\text {th }}$ TRB Annual Meeting, Washington, D.C., January 22-26, 2006.

[7] Institute of Transportation Engineer. Traffic Detector Handbook, 2nd ed.. Washington D.C., 1997.

[8] Shoaib, L. Calibration of FRESIM for Work Zones Using Field Observable Parameters. M.S. Thesis, University of Cincinnati, 2002

[9] T.H. Maze, D.S. Schrock, and A. Kamyab, "Capacity of Freeway Work Zone Lane Closures," In Mid-Continent Transportation Symposium 2000 Proceedings. Iowa State University, Iowa, 2000. 